\title{
Mediation Analyses in Partial Least Squares Structural Equation Modeling, Helping Researchers Discuss More Sophisticated Models: An Abstract
}

\author{
Christian Nitzl, José L. Roldán, and Gabriel Cepeda
}

\begin{abstract}
Indirect or mediated effects constitute a type of relationship between constructs that often occurs in partial least squares structural equation models (PLSSEM). Over the past few years, the methods for testing mediation have become more sophisticated. However, many researchers continue to use outdated methods to test mediation effects in PLS-SEM, which can lead to erroneous results. One reason for the use of outdated methods or even ignoring it is that no systematic tutorials on PLS-SEM exist that draw on the newest statistical findings. This study illustrates the state-of-the-art use of mediation analysis in the context of PLSSEM. It facilitates the adoption of modern procedures in PLS-SEM by challenging the conventional approach to mediation analysis and providing alternatives that are more accurate. In addition, we propose a decision tree and classification of mediation effects. Our recommended approach offers a wide range of testing options that go beyond simple mediation analysis alternatives, helping researchers discuss their studies in a more accurate way.
\end{abstract}

Acknowledgments The authors would like to thank Joseph F. Hair, Christian M. Ringle, and Marko Sarstedt for their fruitful comments and ideas regarding an earlier version of this manuscript.

C. Nitzl $(\bowtie)$

University of the German Federal Armed Forces Munich, Munich, Germany

e-mail: christian.nitzl@unibw.de

J.L. Roldán • G. Cepeda

Universidad de Sevilla, Sevilla, Spain

e-mail: jlroldan@us.es; gabi@us.es 